\title{
Ultrasonographic Changes of Major Salivary Glands in Primary Sjögren's Syndrome
}

\author{
Kyung-Ann Lee ${ }^{1,2}{ }^{\oplus}$, Sang-Heon Lee ${ }^{2}$ and Hae-Rim Kim ${ }^{2, *}$ \\ 1 Division of Rheumatology, Department of Internal Medicine, Soonchunhyang University Seoul hospital, \\ Seoul 04401, Korea; cyberag@naver.com \\ 2 Division of Rheumatology, Department of Internal Medicine, Konkuk University Medical Center, \\ Research institute of medical science, Konkuk University School of Medicine, Seoul 05030, Korea; \\ shlee@kuh.ac.kr \\ * Correspondence: kimhaerim@kuh.ac.kr; Tel.: + 82-2-2030-7542
}

Received: 17 February 2020; Accepted: 13 March 2020; Published: 16 March 2020

\begin{abstract}
We aimed to evaluate the changes over time in salivary gland (SG) abnormalities by ultrasound (US) in patients with primary Sjögren's syndrome (pSS). Patients with pSS $(n=70)$ and idiopathic sicca syndrome $(n=18)$ underwent baseline salivary gland ultrasound (SGUS) scans, and follow-up scans two years later. The semi-quantitative SGUS score (0-48) and intraglandular power Doppler signal (PDS) were assessed. We found that in the pSS group, the SGUS scores for total SGs and bilateral parotid glands significantly increased after the median 23.4-months follow-up. SGUS scores either worsened, improved, or were stable in $18.6 \%, 2.9 \%$, and $78.6 \%$ of patients with pSS, respectively. The median changes from baseline in SGUS scores for total and parotid glands were +1.0 and +0.5 , respectively. None of the SGUS scores changed significantly in the controls. The variables of homogeneity and hypoechoic showed a statistically significant progression of SGUS scores. In pSS patients, the baseline and follow-up PDS scores were significantly higher in the "worsening" group than in the "no change/improvement" group. Overall, the structural abnormalities in major SGs assessed using SGUS remained stable in patients with pSS. At the 2-year follow-up, SGUS scores worsened in $18.6 \%$ of patients with pSS. Intra-glandular hypervascularity was associated with the worsening of SG abnormalities.
\end{abstract}

Keywords: Sjögren's syndrome; salivary glands; ultrasonography; doppler; longitudinal studies

\section{Introduction}

Primary Sjögren's syndrome (pSS) is a systemic autoimmune disease characterized by lymphocytic infiltration of the exocrine glands resulting in dry eye and mouth [1]. Although long known to run a chronic and slowly progressive course in most patients, direct data on the natural history of glandular changes in pSS is insufficient. A few studies with sequential histopathologic evaluations of minor salivary glands (MSG) have been performed in patients with pSS. A 1992 study showed a significant increase in focus score after a mean interval of 3.5 years [2]. In addition, Jonsson et al. demonstrated that focus score increased significantly after mean $39 \pm 20$ months [3]. In contrast, during a median 55-month time interval of repetitive MSG biopsies (MSGB), the histological grades of inflammation in MSG remained almost unchanged [4].

A positive MSGB is a mandatory criterion for the classification of pSS in patients with absence of anti-SS-A/Ro [5]. Whether MSGB could detect a change sensitively, however, is uncertain. And the reproducibility and reliability of MSGB are also issues for its use as a biomarker [6]. Preferring its non-invasiveness and direct visualization of major salivary glands, a large number of studies have reported salivary gland ultrasound (SGUS) as a promising diagnostic tool $[7,8]$. Recently, two trials 
have used SGUS to monitor the therapeutic response to rituximab. These two trials demonstrated the improvement of SGUS scores after treatment with rituximab, suggesting SGUS as an imaging biomarker for pSS $[9,10]$. Despite this promise, evidence on the natural history of SGUS findings in patients with pSS is scarce. Although one study assessed the changes of SGUS over time, the number of patients was limited, and the follow-up SGUS was not performed routinely, leading to an irregular interval between the first and the second SGUS [11].

In this study, we aimed to evaluate the natural course of SGUS after a 2-year interval in patients with pSS and idiopathic sicca syndrome using the SGUS greyscale scoring system and intra-glandular power Doppler US (PDUS). The secondary aim was to assess the predictive factors of progressive changes in major salivary glands based on the SGUS scoring system.

\section{Experimental Section}

\subsection{Study Population}

This was a single-center prospective study performed at Konkuk University Medical Center, Korea, from March 2016 to February 2019. We enrolled patients with pSS and idiopathic sicca syndrome. The definitive diagnosis of pSS was made in accordance with the American-European Consensus (AECG) criteria [12]. Patients who did not fulfil the AECG criteria for pSS and received a diagnosis of idiopathic sicca syndrome, defined as non-immune mediated manifestations of dry eyes and mouth, served as controls. We excluded the patients with secondary Sjogren's syndrome or pSS who received rituximab due to interference in investigating the time-course of SGUS changes. This study was conducted in accordance with the Declaration of Helsinki and approved by the Institutional Review Board for Human Research, Konkuk University Medical Center (KUH 1010776). The written informed consent was obtained from all participants.

\subsection{Salivary Gland Ultrasonography Examination}

The same examiner (L.K.A.) who was unaware of the clinical data performed SGUS on all patients. An HD15 US (Philips Ultrasound, Bothell, WA, USA) device with a linear 5-12 MHz probe was used. The bilateral parotid and submandibular glands were examined in the longitudinal and transverse planes. The patient was supine with the head slightly tilted opposite to the side being scanned to assess the parotid glands. Then the US of the submandibular glands was performed with the head maximally tilted back in a supine position. The thyroid was also scanned for evaluation of parenchymal echogenicity to compare to the salivary glands [13].

The semi-quantitative SGUS score (0-48) developed by Hocevar et al. [14] was used to investigate several aspects: (1) Parenchymal echogenicity compared to thyroid parenchyma, graded 0-1; (2) homogeneity, graded 0-3; (3) presence of hypoechoic areas in parenchyma, graded 0-3; (4) presence of hyperechoic foci, graded 0-3 in parotid glands and 0-1 in submandibular glands; (5) clearness of the salivary gland border, graded $0-3$. The total ultrasound score was the sum of these five domains, ranged from 0 to 48 . Since the cut-off value of 14 reproduced the best diagnostic accuracy in our previous work, [13] a score greater than 14 was defined as abnormal SGUS.

Intra-glandular PDUS was interpreted through a 4-grade semi-quantitative scoring system as follows: Grade $0=$ no parenchymal flow, grade $1=$ up to three single spots signals, or up to two confluent spots, or one confluent spot plus up to two single spots, grade $2=$ flow signals in less than half of the cross-section of a gland $(\leq 50 \%)$, and grade $3=$ flow signals in more than half of the cross-section of a gland $(>50 \%)$. The normal large vessels visible within the salivary glands were excluded from the PDUS score.

Enrolled patients underwent SGUS twice at baseline and 2 years ( \pm 2 months) later. The changes in these SGUS variables were evaluated in patients with pSS and idiopathic sicca syndrome. 


\subsection{Clinical and Laboratory Evaluation}

Standardized clinical and laboratory evaluations were performed. For each patient, the following data were obtained and recorded: Patient demographics, history of dry eyes and mouth, and recurrent parotid enlargement, duration of subjective sicca symptoms, medication use, Schirmer's test result, and whole unstimulated salivary flow rate. Enrolled patients underwent unstimulated salivary flow rate (USFR) tests twice at baseline and 2 years ( \pm 2 months) later, according to standardized methods [15]. To assess the disease activity at baseline, we measured the European League Against Rheumatism Sjögren's syndrome disease activity index (ESSDAI) [16]. All patients completed the European League Against Rheumatism Sjögren's Syndrome Patient-Reported Index (ESSPRI) to evaluate patient-reported dryness, fatigue, and pain [17].

Routine laboratory analyses were conducted at the first SGUS evaluation. Laboratory tests included white blood cell (WBC) count, lymphocyte, hemoglobin, and platelet concentrations, immunoglobulin $\mathrm{G}$ (IgG), and complement levels (C3, C4, CH50). Immunological testing included antinuclear antibody (ANA) (assessed on HEp-2 cells, a titer $\geq 1.160$ was considered positive), anti-SS-A/Ro, and anti-SS-B/La (by enzyme-linked immunosorbent assay (ELISA)), and rheumatoid factor ((RF) by nephelometry) levels. Anti-SS-A/Ro and anti-SS-B/La testings were performed again at the time of follow-up SGUS.

\subsection{Statistical Analysis}

All statistical analyses were performed using the SPSS software package for Windows v. 17.0 (SPSS Inc., Chicago, IL, USA). A Mann-Whitney $U$ and a Chi-squared test analyzed quantitative variables and categorical data, respectively. The SGUS scores between the two time points were compared using the paired Wilcoxon test. The improvement/worsening of SGUS scores was defined as a $\geq 1$-point decrease/increase in the score. To identify SGUS items independently associated with worsening SGUS scores, we performed multivariate linear regression analyses. All SGUS domains that were associated with increased SGUS scores by univariate analysis with $p$-values less than 0.1 were entered into the multivariate model, applying backwards elimination. Results were considered statistically significant when $p<0.05$.

\section{Results}

\subsection{Baseline Characteristics of the Study Population}

In total, 70 patients with pSS and 18 patients with idiopathic sicca syndrome completed the baseline and 2-year follow-up SGUS. Table 1 summarizes the baseline characteristics of the study population. No apparent differences were observed between the two groups regarding age, gender, duration of sicca symptoms, and proportion of patients with subjective and objective sicca symptoms.

Table 1. Baseline characteristics of the study population.

\begin{tabular}{cccc}
\hline & $\begin{array}{c}\text { pSS } \\
(n=70)\end{array}$ & $\begin{array}{c}\text { Idiopathic Sicca } \\
\text { Syndrome } \\
(\boldsymbol{n}=\mathbf{1 8})\end{array}$ & $p$-Value \\
\hline Age, median (IQR), years & $57.6(18.0)$ & $65.5(6.0)$ & 0.053 \\
\hline Female, $n(\%)$ & $67(95.7)$ & $17(94.4)$ & 1.000 \\
\hline Xerostomia, $n(\%)$ & $5.0(7.0)$ & $5.5(7.0)$ & 0.299 \\
\hline Xerophthalmia, $n(\%)$ & $68(67.1)$ & $18(100.0)$ & 1.000 \\
\hline Abnormal Schirmer's test, $n(\%)$ & $65(92.9)$ & $14(77.8)$ & 0.080 \\
\hline USFR, median (IQR), $\mathrm{mL} / 15$ min & $69(98.6)$ & $18(100.0)$ & 1.000 \\
\hline Abnormal USFR, $n(\%)$ & $1.3(2.0)$ & $2.75(2.28)$ & 0.247 \\
\hline Positive ANA, $n(\%)$ & $41(58.6)$ & $6(33.3)$ & 0.056 \\
\hline
\end{tabular}


Table 1. Cont.

\begin{tabular}{cccc}
\hline & $\begin{array}{c}\text { pSS } \\
(n=70)\end{array}$ & $\begin{array}{c}\text { Idiopathic Sicca } \\
\text { Syndrome } \\
(\boldsymbol{n}=\mathbf{1 8})\end{array}$ & $p$-Value \\
\hline Positive anti-Ro/SSA, $n(\%)$ & $64(91.4)$ & $0(0)$ & $<0.001$ \\
\hline Positive anti-La/SSB, $n(\%)$ & $35(50.0)$ & $0(0)$ & $<0.001$ \\
\hline Positive RF, $n(\%)$ & $31(44.3)$ & $7(38.9)$ & 0.743 \\
\hline RF, median (IQR), IU/dL & $27.0(31)$ & $15.0(32)$ & 0.392 \\
\hline IgG, median (IQR), $\mathrm{mg} / \mathrm{dL}$ & $1801.0(440)$ & $1546.1(485)$ & 0.010 \\
\hline C3, median (IQR), $\mathrm{mg} / \mathrm{dL}$ & $94.5(9.3)$ & $98.8(23.8)$ & 0.011 \\
\hline C4, median (IQR), mg/dL & $25.1(10.0)$ & $23.0(9.0)$ & 0.108 \\
\hline Total SGUS scores at baseline, median (IQR) & $27(14)$ & $4(3)$ & $<0.001$ \\
\hline Total SGUS scores $\geq 14$ at baseline, $n$ (\%) & $55(78.6)$ & $1(5.6)$ & $<0.001$ \\
\hline Total SGUS scores for the parotid glands at baseline, median (IQR) & $12.0(10)$ & $2.0(2)$ & $<0.001$ \\
\hline Total SGUS scores for the submandibular glands at baseline, median & $14.0(9)$ & $2.0(4)$ & $<0.001$ \\
\hline PDS sum scores of four salivary glands, median (IQR) & $0(3)$ & $1(3)$ & 0.320 \\
\hline
\end{tabular}

pSS: Primary Sjögren's syndrome; IQR: Interquartile range; USFR: Unstimulated salivary flow rate; ANA: Antinuclear antibody; SSA: Sjögren's-syndrome-related antigen A, SSB: Sjögren's-syndrome-related antigen B; RF: Rheumatoid factor; Ig: Immunoglobulin; C: Complement; SGUS: Salivary gland ultrasound; PDS: Power Doppler signal.

As expected, all SGUS scores were higher in patients with $\mathrm{pSS}$ compared to patients with idiopathic sicca syndrome $(p<0.001)$. There were no significant differences between the two groups concerning the PDS sum scores of four salivary glands.

\subsection{Changes in SGUS Scores over Time}

Patients with pSS had shorter median interval time between the first and the second SGUS compared to patients with idiopathic sicca syndrome (median (IQR); 23.4 (2.7) vs. 25.4 (4.2) months) $(p<0.002)$. In the pSS group, the total SGUS scores and the SGUS scores for bilateral parotid glands significantly increased during the median 23.4-month follow-up ( $p=0.013$ and $p=0.011$, respectively) (Table 2) (Figure 1). At follow-up, the absolute changes from baseline in total SGUS scores and SGUS scores for bilateral parotid glands in the pSS group were +1.0 and +0.5 , respectively. The domains of homogeneity and hypoechoic areas showed a statistically significant progression of SGUS scores ( $\beta=1.036, p<0.001$ and $\beta=0.736, p=0.004$, respectively). None of the SGUS scores changed significantly in patients with idiopathic sicca syndrome. The PDS sum scores of four salivary glands and USFR did not significantly change.
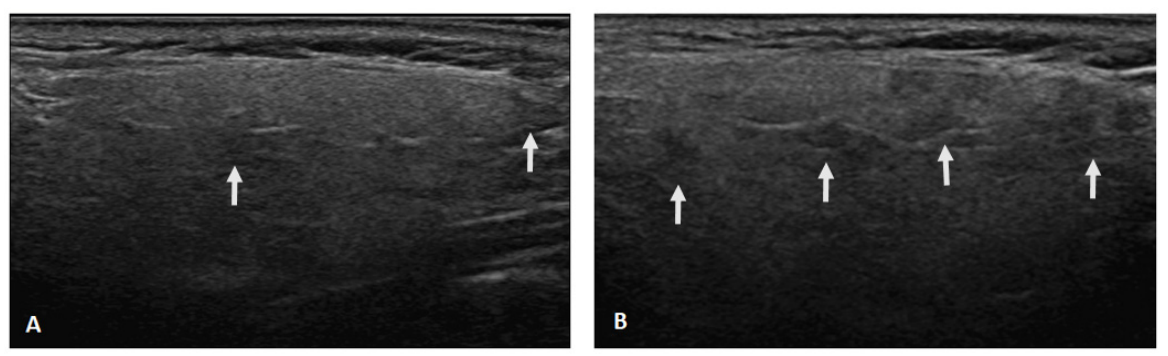

Figure 1. Ultrasonographic changes of the parotid gland after a 2-year follow-up. (A) The presence of hypoechoic areas (arrows) in the parenchyma increased from grade 1 (defined as few scattered) (A) to grade 2 (defined as several) $(\mathbf{B})$. 
Table 2. Changes in SGUS scores over time.

\begin{tabular}{cccccccc}
\hline & & $\begin{array}{c}\text { pSS } \\
(n=70)\end{array}$ & & \multicolumn{2}{c}{$\begin{array}{c}\text { Idiopathic Sicca Syndrome } \\
(n=18)\end{array}$} & $\begin{array}{c}\text { pSS vs. Idiopathic } \\
\text { Sicca Syndrome at } \\
\text { Follow-Up }\end{array}$ \\
\hline & Baseline & Follow-up & $p$-Value & Baseline & Follow-up & $p$-value & $p$-value \\
\hline Total SGUS scores & $27(14)$ & $28(15)$ & 0.013 & $4(3)$ & $4.5(4)$ & 0.157 & $<0.001$ \\
\hline $\begin{array}{c}\text { Total SGUS scores } \geq 14 \text { at } \\
\text { baseline, } n \text { (\%) }\end{array}$ & $55(78.6)$ & $56(80.0)$ & 1.000 & $1(5.6)$ & $1(5.6)$ & 1.000 & $<0.001$ \\
\hline $\begin{array}{c}\text { SGUS scores for the } \\
\text { parotid glands }\end{array}$ & $12(10)$ & $12(9)$ & 0.011 & $2(2)$ & $2(2)$ & 1.000 & $<0.001$ \\
\hline $\begin{array}{c}\text { SGUS scores for the } \\
\text { submandibular glands }\end{array}$ & $14(9)$ & $14(8)$ & 0.154 & $2(4)$ & $2(4)$ & 0.157 & $<0.001$ \\
\hline $\begin{array}{c}\text { PDS sum scores of four } \\
\text { salivary glands }\end{array}$ & $0(3)$ & $0(3)$ & 1.000 & $2(3)$ & $2(2)$ & 0.786 & 0.303 \\
\hline $\begin{array}{c}\text { USFR, mL/15 min } \\
\text { (1) }\end{array}$ & $1.3(2.0)$ & $1.0(1.67)$ & 0.086 & $\begin{array}{c}2.75 \\
(2.28)\end{array}$ & $2.5(6.9)$ & 0.285 & 0.073 \\
\hline
\end{tabular}

Data are expressed as median (IQR). $p$-values in bold are significant. pSS: Primary Sjögren's syndrome; IQR: Interquartile range; SGUS: Salivary gland ultrasound; PDS: Power Doppler signal, USFR: Unstimulated salivary flow rate.

\subsection{Comparison of Clinical, Laboratory, and SGUS Features in Relation to SGUS Scores}

We separated the patients into two groups based on whether SGUS scores worsened $(n=13)$, improved $(n=2)$, or showed no change $(n=55)$. Baseline and follow-up PDS sum scores of four salivary glands were significantly higher in the worsening SGUS group than no change/improvement SGUS group. No significant differences were observed between the two groups regarding age, duration of sicca symptoms, medication, serologic markers, USFR, ESSPRI, and ESSDAI (Table 3).

Table 3. Clinical, laboratory, and SGUS features according to changes in SGUS scores.

\begin{tabular}{cccc}
\hline & $\begin{array}{c}\text { Worsening } \\
(\boldsymbol{n}=\mathbf{1 3})\end{array}$ & $\begin{array}{c}\text { No Change or } \\
\text { Improved } \\
(\boldsymbol{n}=\mathbf{5 5 / 2})\end{array}$ & $p$-Value \\
\hline Age, years & $62.0(16)$ & $57.0(19)$ & 0.197 \\
\hline Female, $n(\%)$ & $13(100)$ & $54(94.7)$ & 1.000 \\
\hline Duration of sicca symptoms, years & $5.0(8)$ & $5.0(6.0)$ & 0.058 \\
\hline Interval from 1st to 2nd SGUS, months & $24.2(2.4)$ & $23.0(2.8)$ & 0.369 \\
\hline Xerostomia, $n(\%)$ & $13(100)$ & $55(96.5)$ & 1.000 \\
\hline Xerophthalmia, $n(\%)$ & $12(92.3)$ & $53(93.0)$ & 1.000 \\
\hline Abnormal Schirmer's test, $n(\%)$ & $13(100)$ & $56(98.2)$ & 1.000 \\
\hline Baseline USFR, mL/15 min & $2.2(2.7)$ & $1.3(2.0)$ & 0.932 \\
\hline Follow-up USFR, mL/15 min & $3.0(3.25)$ & $1.0(1.5)$ & 0.606 \\
\hline Current medication & & & 0.720 \\
\hline Hydroxychloroquine $(\mathrm{HCQ}), n(\%)$ & $11(84.6)$ & $44(77.2)$ & 0.877 \\
\hline HCQ dose, $\mathrm{mg} / \mathrm{day}$ & $200(150)$ & $200(200)$ & 1.000 \\
\hline Corticosteroids $n(\%)$ & $3(23.1)$ & $12(21.1)$ & 0.950 \\
\hline Dose of corticosteroids, mg/day & $0(1.25)$ & $0(0)$ & 1.000 \\
\hline Azathioprine, $n(\%)$ & $1(7.7)$ & $6(10.5)$ & 0.091 \\
\hline Positive ANA, $n(\%)$ & $12(92.3)$ & $38(66.67)$ & 0.308 \\
\hline Positive anti-Ro/SSA, $n(\%)$ & $11(84.6)$ & $53(93.0)$ & 0.540 \\
\hline Positive anti-La/SSB, $n(\%)$ & $8(61.5)$ & $27(47.4)$ & \\
\hline
\end{tabular}


Table 3. Cont.

\begin{tabular}{|c|c|c|c|}
\hline & $\begin{array}{l}\text { Worsening } \\
(n=13)\end{array}$ & $\begin{array}{c}\text { No Change or } \\
\text { Improved } \\
(n=55 / 2)\end{array}$ & $p$-Value \\
\hline Negative seroconversion anti-Ro/SSA at follow-up, $n(\%)$ & $1(7.7)$ & $9(15.8)$ & 0.675 \\
\hline Negative seroconversion of anti-La/SSA at follow-up, $n(\%)$ & $1(7.7)$ & $5(8.8)$ & 1.000 \\
\hline Positive seroconversion of anti-Ro/SSA at follow-up, $n(\%)$ & $0(0)$ & $0(0)$ & - \\
\hline Positive seroconversion of anti-La/SSA at follow-up, $n(\%)$ & $0(0)$ & $1(1.8)$ & 1.000 \\
\hline $\mathrm{RF}, \mathrm{IU} / \mathrm{dL}$ & $15(31)$ & $27(30)$ & 0.907 \\
\hline $\mathrm{IgG}, \mathrm{mg} / \mathrm{dL}$ & $1948(607)$ & $1546(499)$ & 0.107 \\
\hline $\mathrm{C} 3, \mathrm{mg} / \mathrm{dL}$ & $92.8(7.0)$ & $97.6(26.1)$ & 0.123 \\
\hline $\mathrm{C} 4, \mathrm{mg} / \mathrm{dL}$ & $20.3(9.3)$ & $23.0(8.7)$ & 0.874 \\
\hline ESSDAI (123 = maximal disease activity) & $4.0(4.0)$ & $2.0(3.0)$ & 0.225 \\
\hline ESSPRI (10 = maximal symptom severity) & $6(4.35)$ & $5.7(3.3)$ & 0.606 \\
\hline Total SGUS scores at baseline & $24(12)$ & $29(15)$ & 0.634 \\
\hline Total SGUS scores $\geq 14$ at baseline, $n(\%)$ & $11(84.6)$ & $44(77.2)$ & 0.720 \\
\hline Total SGUS scores at follow-up & $27(11)$ & $28(15)$ & 0.487 \\
\hline Total SGUS scores $\geq 14$ at follow-up, $n(\%)$ & $12(92.3)$ & $44(77.2)$ & 0.441 \\
\hline Total SGUS scores for the parotid glands at baseline & $11(7)$ & $12(11)$ & 0.838 \\
\hline Total SGUS scores for the submandibular glands at baseline & $14(7)$ & $14(10)$ & 0.617 \\
\hline Total SGUS scores for the parotid glands at follow-up & $13(6)$ & $12(11)$ & 0.57 \\
\hline Total SGUS scores for the submandibular glands at follow-up & $16(4)$ & $14(10)$ & 0.585 \\
\hline Baseline PDS sum scores of four salivary glands & $2(3)$ & $0(2)$ & 0.008 \\
\hline Follow-up PDS sum scores of four salivary glands & $2(3)$ & $0(2)$ & 0.008 \\
\hline \multicolumn{4}{|c|}{$\begin{array}{l}\text { Value are presented as median (IQR) unless otherwise stated. } p \text {-values in bold are statistically significant. SGUS: } \\
\text { Salivary gland ultrasound; pSS: Primary Sjögren's syndrome; IQR: Interquartile range; USFR: Unstimulated salivary } \\
\text { flow rate; ANA: Antinuclear antibody; SSA: Sjögren's-syndrome-related antigen A, SSB: Sjögren's-syndrome-related } \\
\text { antigen B; RF: Rheumatoid factor; Ig: Immunoglobulin; C: Complement; ESSDAI: European League Against } \\
\text { Rheumatism Sjögren's Syndrome Disease Activity Index; ESSPRI: European Sjögren's Syndrome Patient Reported } \\
\text { Index; PDS: Power Doppler signal. }\end{array}$} \\
\hline
\end{tabular}

In the pSS group, 15 patients had an SGUS score $<14$ at baseline. Among them, SGUS scores changed in 2 patients (13.3\%) after the follow-up study. One out of the 15 initially negative SGUS (SGUS score 13) was positive on the second US study (SGUS score 16). Another patient with increased SGUS scores (from 7 to 10) had negative SGUS in the initial and secondary US exams.

\section{Discussion}

The present longitudinal ultrasound study demonstrated the progressive course of major salivary glands in patients with pSS. Moreover, SGUS scores did not change significantly over 2 years in patients with idiopathic sicca syndrome. In contrast to our study, a recent study showed that SGUS abnormalities did not change significantly in both pSS and non-pSS patients during a mean of 1.9 years. However, the second SGUS was not a routine evaluation but was instead part of standard care by the managing physician, leading to follow-up intervals ranging from 1 year to 7 years. To avoid this variation, we investigated the SGUS scores at baseline then 2 years ( \pm 2 months) later to confirm the natural course of major salivary glands. In the pSS group, salivary glandular involvement remained stable in a majority of patients, but $18.6 \%$ of patients had worsening SGUS scores, and the median absolute total SGUS scores changed 1.0 during the 2 years. Our findings suggested a slowly progressive course of salivary glandular involvement in patients with pSS.

We defined improvement or worsening of SGUS scores as a $\geq 1$-point change in the score. Two randomized controlled trials also used SGUS for monitoring of the therapeutic response 
of rituximab in patients with pSS. Similar to our study, both trials also defined improvement in echostructure as a $\geq 1$-point decrease in the score after 24 and 48 weeks of treatment $[9,10]$. However, one-point changes in SGUS scores could be marginal and insufficient to provide clinical significance. Therefore, longer longitudinal studies are needed to reveal sensitivity to changes in SGUS scores in patients with pSS.

To date, a diversity of SGUS scoring systems have been developed, aiming to improve the diagnosis of pSS. The most relevant SGUS feature is parenchymal inhomogeneity [18]. However, there are other detectable SGUS abnormalities, such as decreased echogenicity, hyperechoic bands, calcifications, size variations of hypo-anechoic areas, intraglandular lymph nodes, irregularities of glandular borders, and hypervascularity [19]. Since we assumed that only evaluating the homogeneity and hypoechoic areas could be insufficient to reflect the time course of SGUS in pSS, the present study used the semi-quantitative SGUS score (0-48). Five domains comprise the $0-48$ SGUS scoring system, including not only homogeneity and hypoechogenic areas, but also parenchymal echogenicity, clearness of posterior border margin, and hyperechogenic reflections [14]. We examined which domains were associated with the progression of SGUS scores. We found significant changes in inhomogeneity and hypoechoic areas over 2 years, which are US hallmarks of pSS. Changes in the other SGUS domains, however, were not observed. The two-year follow-up period could be a relatively short period to describe the whole longitudinal features of SGUS in pSS. Moreover, the corresponding histopathologic features of each SGUS variable are unknown. Which US finding represents irreversible (damage), or reversible (inflammation) findings remain questionable [18]. Therefore, studies integrating US and histopathology of salivary glands are needed to determine the sensitive markers of SGUS over time.

The power Doppler (PD) signals represent the status of increased blood flow and active inflammation. Although increased PD signals of synovitis were reported to be associated with radiographic progression in rheumatoid arthritis [20], evidence suggesting a connection between increased PD signals and prognosis of salivary glands is lacking in pSS. We found that PSS patients with worsening SGUS scores had higher baseline and follow-up PDUS scores compared to patients with no change or improvement of SGUS scores. In our previous work, we described reduced PDUS scores in patients with advanced pSS compared to pSS patients without definite SGUS structural abnormality, suggesting that hypervascularity reflecting the inflammatory phase of pSS could lead to glandular hypovascularity in late stages of pSS [13]. Together, our present and previous studies indicate that intraglandular hypervascularity could result in the structural progression of salivary glands in patients with pSS. However, without explicit data directly correlating intra-glandular PD signals with active inflammation, further studies comparing histopathologic findings (vascularity and inflammation) and PDUS are needed. In addition, this study showed that the PDS scores in the worsening group did not change at the follow-up when the median value of SGUS scores became comparable to those of the no change/improved group. Considering the slowly progressive course of pSS, glandular hypervascularization in the inflammatory phase could be unaltered and last more than 2 years. A long-term longitudinal study evaluating changes in PDS scores of salivary glands is needed to reveal the duration of glandular vascularity and confirm the link between hypervascularity and progression of parenchymal abnormalities of salivary glands.

To assess the factors associated with glandular progression in patients with pSS, various clinical, laboratory, and SGUS features were compared between worsening and no change/improved SGUS groups. However, prognostic factors for pSS, such positive autoantibody titers, levels of complement, and ESSDAI were not different between the two groups. Only PDS sum scores showed a significant difference between the two groups. Although well-designed randomized, controlled trials (RCT) providing evidence for the role of Hydroxychloroquine (HCQ) in pSS are insufficient, current published studies showed that the efficacy of HCQ in pSS was not superior to placebo in treating dry mouth or dry eyes in pSS patients [21]. Corroborating this, one RCT showed no significant difference in USFR between patients treated with HCQ and placebo [22]. Inconsistent with the previous studies, we also 
confirmed that the progression of major salivary glands in pSS measured by SGUS scores was not associated with the use of HCQ.

The prevalence of anti-Ro/SSA is 50-70\% in pSS, and anti-Ro/SSA and anti-La/SSB correlate with younger age at diagnosis, more severe dysfunction of the exocrine gland, and higher intensity of the exocrine glands [23]. In our previous work, double seropositivity of anti-Ro/SSA and La/SSB was independently associated with high SGUS scores in pSS [13]. Utilizing this previously-published data, we further examined the changes in anti-Ro/SSA and La/SSB after 2 years to assess the association with progressive glandular involvement of pSS. No association between seroconversion and changes of SGUS scores was found. Negative seroconversion of anti-Ro/SSA and anti-La/SSB were observed in $14.2 \%$ and $8.6 \%$ of patients with pSS, respectively. Positive seroconversion of anti-Ro/SSA and anti-La/SSB was rarely observed ( $0 \%$ and $1.4 \%$, respectively). The frequent fluctuation of anti-extractable antigens (anti-ENA) in systemic lupus erythematosus was reported in a longitudinal study, showing $30-50 \%$ of negative and $14.7-56 \%$ of positive seroconversion of anti-ENA [24]. Compared to the SLE study, we found less anti-Ro/SSA and anti-La/SSB fluctuations over time in patients with pSS.

We observed that USFR did not change significantly in both pSS and idiopathic sicca syndrome groups. A previous study reported that stimulated salivary flow rates (SSFR) significantly dropped at a mean of 3.6 years of follow up in patients with pSS, although no alteration of USFR was observed [25]. On the other hand, Jonsson et al. described a mean follow-up of 39 months with 21 pSS patients and showed no reduction in stimulated whole salivary secretion [3]. Salivary gland dysfunction is a key manifestation of pSS and evaluation of USFR is a diagnostic criterion for pSS [5]. However, USFR showed low sensitivity (52\%) in establishing the diagnosis of pSS [26], and the USFR test is relatively dependent on drug effects and environmental factors compared to SSFR [3]. The weak test-retest reliability of USFR has been reported [27]. Therefore, the USFR may not be suitable for monitoring glandular function. Further studies using both USFR and SSFR are needed to investigate the long-term natural course of salivary gland function in patients with pSS.

While providing insight into pSS progression, the present study has some limitations. The number of patients with idiopathic sicca syndrome was relatively small, and the data on changes in SGUS scores in healthy controls without dry mouth and SSFR were absent. Furthermore, this study was a single-center study and a single expert performed SGUS at a 2-year follow up. Although the homogeneity item showed good inter-observer reliability [28], we could not calculate the inter-observer agreement for each item in this study. Since SGUS scores barely worsened over a short follow up, long-term multicenter studies with a reliability exercise are needed to verify our study results and reveal the meaning of changes in SGUS scores.

\section{Conclusions}

In conclusion, the structural abnormalities in major salivary glands assessed using SGUS scores remained stable in the majority of patients with pSS. During the 2-year study, SGUS scores progressed in $18.6 \%$ of patients with pSS. The homogeneity and hypoechoic areas are domains that showed significant progression. Intra-glandular hypervascularity was associated with the worsening of salivary gland abnormalities, providing a potential predictive marker for glandular progression in pSS.

Author Contributions: Conceptualization, K.-A.L. and H.-R.K.; methodology, K.-A.L.; software, K.-A.L.; validation, S.-H.L.; formal analysis, K.-A.L. and H.-R.K.; investigation, K.-A.L. and S.-H.L.; resources, S.-H.L.; data curation, S.-H.L.; writing-original draft preparation, K.-A.L. writing-review and editing, K.-A.L. and H.-R.K.; visualization, S.-H.L.; supervision, S.-H.L.; project administration, H.-R.K.; funding acquisition, H.-R.K. All authors have read and agreed to the published version of the manuscript.

Funding: This work was funded by the Konkuk University Medical Center Research Grant 2019.

Acknowledgments: In this section you can acknowledge any support given which is not covered by the author contribution or funding sections. This may include administrative and technical support, or donations in kind (e.g., materials used for experiments).

Conflicts of Interest: The authors declare that they have no conflict of interest. 


\section{References}

1. Mariette, X.; Criswell, L.A. Primary Sjogren's Syndrome. N. Engl. J. Med. 2018, 378, 931-939. [CrossRef] [PubMed]

2. Leroy, J.P.; Pennec, Y.L.; Soulier, C.; Berthelot, J.M.; Letoux, G.; Youinou, P. Follow up study of labial salivary gland lesions in primary Sjogren's syndrome. Ann. Rheum. Dis. 1992, 51, 777-780. [CrossRef] [PubMed]

3. Jonsson, R.; Kroneld, U.; Backman, K.; Magnusson, B.; Tarkowski, A. Progression of sialadenitis in Sjogren's syndrome. Br. J. Rheumatol. 1993, 32, 578-581. [CrossRef] [PubMed]

4. Kapsogeorgou, E.K.; Christodoulou, M.I.; Panagiotakos, D.B.; Paikos, S.; Tassidou, A.; Tzioufas, A.G.; Moutsopoulos, H.M. Minor salivary gland inflammatory lesions in Sjogren syndrome: Do they evolve? J. Rheumatol. 2013, 40, 1566-1571. [CrossRef]

5. Shiboski, C.H.; Shiboski, S.C.; Seror, R.; Criswell, L.A.; Labetoulle, M.; Lietman, T.M.; Rasmussen, A.; Scofield, H.; Vitali, C.; Bowman, S.J.; et al. 2016 American College of Rheumatology/European League Against Rheumatism classification criteria for primary Sjogren's syndrome: A consensus and data-driven methodology involving three international patient cohorts. Ann. Rheum. Dis. 2017, 76, 9-16. [CrossRef]

6. Fisher, B.A.; Brown, R.M.; Bowman, S.J.; Barone, F. A review of salivary gland histopathology in primary Sjogren's syndrome with a focus on its potential as a clinical trials biomarker. Ann. Rheum. Dis. 2015, 74, 1645-1650. [CrossRef]

7. Delli, K.; Dijkstra, P.U.; Stel, A.J.; Bootsma, H.; Vissink, A.; Spijkervet, F.K. Diagnostic properties of ultrasound of major salivary glands in Sjogren's syndrome: A meta-analysis. Oral Dis. 2015, 21, 792-800. [CrossRef]

8. Mossel, E.; Delli, K.; van Nimwegen, J.F.; Stel, A.J.; Kroese, F.G.M.; Spijkervet, F.K.L.; Vissink, A.; Arends, S.; Bootsma, H. Ultrasonography of major salivary glands compared with parotid and labial gland biopsy and classification criteria in patients with clinically suspected primary Sjogren's syndrome. Ann. Rheum. Dis. 2017, 76, 1883-1889. [CrossRef]

9. Fisher, B.A.; Everett, C.C.; Rout, J.; O’Dwyer, J.L.; Emery, P.; Pitzalis, C.; Ng, W.F.; Carr, A.; Pease, C.T.; Price, E.J.; et al. Effect of rituximab on a salivary gland ultrasound score in primary Sjogren's syndrome: Results of the TRACTISS randomised double-blind multicentre substudy. Ann. Rheum. Dis. 2018, 77, 412-416. [CrossRef]

10. Jousse-Joulin, S.; Devauchelle-Pensec, V.; Cornec, D.; Marhadour, T.; Bressollette, L.; Gestin, S.; Pers, J.O.; Nowak, E.; Saraux, A. Brief Report: Ultrasonographic Assessment of Salivary Gland Response to Rituximab in Primary Sjogren's Syndrome. Arthritis Rheumatol. 2015, 67, 1623-1628. [CrossRef]

11. Gazeau, P.; Cornec, D.; Jousse-Joulin, S.; Guellec, D.; Saraux, A.; Devauchelle-Pensec, V. Time-course of ultrasound abnormalities of major salivary glands in suspected Sjogren's syndrome. Joint Bone Spine 2018, 85, 227-232. [CrossRef] [PubMed]

12. Vitali, C.; Bombardieri, S.; Jonsson, R.; Moutsopoulos, H.M.; Alexander, E.L.; Carsons, S.E.; Daniels, T.E.; Fox, P.C.; Fox, R.I.; Kassan, S.S.; et al. Classification criteria for Sjogren's syndrome: A revised version of the European criteria proposed by the American-European Consensus Group. Ann. Rheum. Dis. 2002, 61, 554-558. [CrossRef] [PubMed]

13. Lee, K.A.; Lee, S.H.; Kim, H.R. Diagnostic and predictive evaluation using salivary gland ultrasonography in primary Sjogren's syndrome. Clin. Exp. Rheumatol. 2018, 36, 165-172. [PubMed]

14. Hocevar, A.; Ambrozic, A.; Rozman, B.; Kveder, T.; Tomsic, M. Ultrasonographic changes of major salivary glands in primary Sjogren's syndrome. Diagnostic value of a novel scoring system. Rheumatology 2005, 44, 768-772. [CrossRef]

15. Navazesh, M.; Kumar, S.K. Measuring salivary flow: Challenges and opportunities. J. Am. Dent. Assoc. 2008, 139, 35s-40s. [CrossRef]

16. Seror, R.; Bowman, S.J.; Brito-Zeron, P.; Theander, E.; Bootsma, H.; Tzioufas, A.; Gottenberg, J.E.; Ramos-Casals, M.; Dorner, T.; Ravaud, P.; et al. EULAR Sjogren's syndrome disease activity index (ESSDAI): A user guide. RMD Open 2015, 1, e000022. [CrossRef]

17. Seror, R.; Ravaud, P.; Mariette, X.; Bootsma, H.; Theander, E.; Hansen, A.; Ramos-Casals, M.; Dorner, T.; Bombardieri, S.; Hachulla, E.; et al. EULAR Sjogren's Syndrome Patient Reported Index (ESSPRI): Development of a consensus patient index for primary Sjogren's syndrome. Ann. Rheum. Dis. 2011, 70, 968-972. [CrossRef] 
18. Luciano, N.; Ferro, F.; Bombardieri, S.; Baldini, C. Advances in salivary gland ultrasonography in primary Sjogren's syndrome. Clin. Exp. Rheumatol. 2018, 36, 159-164.

19. Carotti, M.; Salaffi, F.; Di Carlo, M.; Barile, A.; Giovagnoni, A. Diagnostic value of major salivary gland ultrasonography in primary Sjogren's syndrome: The role of grey-scale and colour/power Doppler sonography. Gland Surg. 2019, 8, S159-S167. [CrossRef]

20. Bhasin, S.; Cheung, P.P. The Role of Power Doppler Ultrasonography as Disease Activity Marker in Rheumatoid Arthritis. Dis. Markers 2015, 2015. [CrossRef]

21. Wang, S.Q.; Zhang, L.W.; Wei, P.; Hua, H. Is hydroxychloroquine effective in treating primary Sjogren's syndrome: A systematic review and meta-analysis. BMC Musculoskelet. Disord. 2017. [CrossRef] [PubMed]

22. Gottenberg, J.E.; Ravaud, P.; Puechal, X.; Le Guern, V.; Sibilia, J.; Goeb, V.; Larroche, C.; Dubost, J.J.; Rist, S.; Saraux, A.; et al. Effects of hydroxychloroquine on symptomatic improvement in primary Sjogren syndrome: The JOQUER randomized clinical trial. JAMA 2014, 312, 249-258. [CrossRef] [PubMed]

23. Fayyaz, A.; Kurien, B.T.; Scofield, R.H. Autoantibodies in Sjogren's Syndrome. Rheum. Dis. Clin. North Am. 2016, 42, 419-434. [CrossRef] [PubMed]

24. Faria, A.C.; Barcellos, K.S.; Andrade, L.E. Longitudinal fluctuation of antibodies to extractable nuclear antigens in systemic lupus erythematosus. J. Rheumatol. 2005, 32, 1267-1272. [PubMed]

25. Pijpe, J.; Kalk, W.W.; Bootsma, H.; Spijkervet, F.K.; Kallenberg, C.G.; Vissink, A. Progression of salivary gland dysfunction in patients with Sjogren's syndrome. Ann. Rheum. Dis. 2007, 66, 107-112. [CrossRef] [PubMed]

26. Speight, P.M.; Kaul, A.; Melsom, R.D. Measurement of whole unstimulated salivary flow in the diagnosis of Sjogren's syndrome. Ann. Rheum. Dis. 1992, 51, 499-502. [CrossRef] [PubMed]

27. Varoni, E.M.; Federighi, V.; Decani, S.; Carrassi, A.; Lodi, G.; Sardella, A. The effect of clinical setting on the unstimulated salivary flow rate. Arch. Oral Biol. 2016, 69, 7-12. [CrossRef]

28. Milic, V.D.; Petrovic, R.R.; Boricic, I.V.; Radunovic, G.L.; Pejnovic, N.N.; Soldatovic, I.; Damjanov, N.S. Major salivary gland sonography in Sjogren's syndrome: Diagnostic value of a novel ultrasonography score (0-12) for parenchymal inhomogeneity. Scand. J. Rheumatol. 2010, 39, 160-166. [CrossRef]

(C) 2020 by the authors. Licensee MDPI, Basel, Switzerland. This article is an open access article distributed under the terms and conditions of the Creative Commons Attribution (CC BY) license (http://creativecommons.org/licenses/by/4.0/). 